\title{
Comparative Study between Submucosal Diathermy and Endoscopic Partial Turbinectomy in Hypertrophied Inferior Turbinate
}

\author{
*Wael Hassan Aboulwafa *, Abd Elaziz Kamal Saad *, Ayman Yehia Abbas*, Mohammed Abed \\ Elewa, **Tarek Abd Elkarem Eldahshan
}

* Otolaryngology dept., ${ }^{* *}$ Clinical Pathology dept., Faculty of Medicine, AL-Azhar University

Corresponding author: Mohammed Abed Elewa, email:drmohamedabed@gmail.com, +201063475773

\begin{abstract}
Background: Chronic nasal obstruction is a common subjective complaint seen by otolaryngologists; turbinate hypertrophy is one of its causes. There are various treatment options for enlarged turbinates, the method of treatment depends on the size of the enlarged turbinates.

Objective: To evaluate the effectiveness of submucosal diathermy of inferior turbinate versus endoscopic partial turbinectomy in patients with inferior turbinate hypertrophy.

Patients and methods: A total of sixty patients of different age groups and both sexes were involved in the study. Patients were divided in two groups; group (I), which included 30 patients, had submucosal diathermy of both inferior turbinates and group (II); which included 30 patients had surgical endoscopic partial turbinectomy of both inferior turbinates. Patients were followed up for 6 months postoperatively and nasal obstruction was analyzed according to (NOSE) scale.

Results: there were no significant differences between both groups in nasal score mean at pre-operative, 1 week and 1 month (post-operative), however, group (II) had significantly ( $<<0.01)$ lower nasal score mean at 6 months compared to group (I),. In addition, there were a significant post-operative improvement in nasal obstruction in both used techniques at 1 week and 1 month. However, this significant improvement continued to 6 months in group II While, a type of recurrence was happened in group I at 6 months.

Conclusion: both of the used surgical techniques were found to be effective in reducing nasal obstruction but endoscopic partial turbinectomy was more effective in reducing nasal obstruction for longer duration.
\end{abstract}

KEYWORDS: Submucosal Diathermy, Endoscopic Turbinectomy,HypertrophyInferior Turbinate

\section{INTRODUCTION}

The inferior turbinates are bony and soft tissue projections that protrude into the nasal passages and play an important role in normal respiratory function, as the main regulator of nasal airflow in which the mucosa of the turbinates is essential for maintaining normal defense, humidification, warming and filtering of inspired air ${ }^{(1)}$. Chronic nasal obstruction is one of the most common human problems and a very frequent symptom in the otorhinolaryngology field ${ }^{(2)}$. It is commonly defined as patient discomfort manifested as a sensation of insufficient airflow through the nose. The etiology of nasal obstruction is generally divided into mucosal and anatomical causes ${ }^{(3)}$.

In some patients, the nasal turbinate remains persistently enlarged or swollen and obstructs the airway to cause a congested feeling and these enlarged inferior turbinates narrow the nasal cavity and contribute significantly to nasal airway obstruction ${ }^{(4)}$. Hypertrophy of the inferior turbinate is the most frequent cause of chronic nasal obstruction and may be related to allergy, pseudo allergy, non allergic rhinitis with eosinophilia syndrome and iatrogenic rhinopathy ${ }^{(5)}$. Mucosal swelling of the inferior turbinates is a part of the physiologic vascular changes which take place during the nasal cycle. Hyper-reactivity, infection and allergy may enhance these changes ${ }^{(6)}$. Enlargement of the inferior turbinate can be due to either an enlargement of the osseous or mucosal component of the turbinate ${ }^{(7)}$.

Medical treatment including antihistamines, topical and systemic steroids, desensitization, and allergen avoidance is used but if these measures fail, surgery is advised ${ }^{(8)}$. There are numerous available surgical techniques in use to address inferior turbinate hypertrophy, 
including turbinectomy, turbinoplasty, extramucosal or submucosal electrocautery, radiofrequency ablation, laser-assisted resection or ablation, and cryosurgery ${ }^{(9)}$. The physiological basis on which reduction of inferior turbinate rests is the Poiseulle's Law which states that the laminar flow rate of air along a pipe is proportional to the fourth power of its radius and hence a small change in the inferior turbinate will dramatically affect the nasal airflow ${ }^{(10)}$. Surgical intervention improves the dynamic competence of the nasal airway by addressing the fixed obstruction and alleviation of symptoms by reducing the effects of edematous mucosa ${ }^{(11)}$.

The goal of turbinate surgery is to obtain an improvement of nasal breathing with preservation of the physiological function of the turbinate and minimal discomfort or adverse effects ${ }^{(12)}$. The majority of surgical techniques provide satisfactory results but adverse events are frequently observed as bleeding, crusting, dryness, foul odor, pain, hyposomia, synechia and bone necrosis ${ }^{(13)}$. Turbinectomy involves removal of all or portion of the inferior turbinate and may be performed by direct visualization or with aid of an endoscope ${ }^{(14)}$. Submucous Diathermy (SMD) involves passing a probe just below the mucosal surface lining of the turbinate bones and cauterizing using heat energy to shrink the size of theses structures, this preserves most of the mucosal lining and allows for preservation of normal function $^{(15)}$. The aim of this work is to evaluate the effectiveness of submucosal diathermy of inferior turbinate versus endoscopic partial turbinectomy in patients with inferior turbinate hypertrophy.

\section{PATIENTS AND METHODS}

This is a prospective comparative study that was carried out at the department of Otorhinolaryngology, Hearing and Speech Institute from March, 2016 to June, 2017 to evaluate the effects of Sub-mucosal diathermy SMD of inferior turbinate versus partial surgical inferior turbinectomy PSIT in patients with chronic hypertrophic rhinitis causing nasal obstruction. The study was approved by the Ethics Board of Al-Azhar University.

A total of sixty patients of different age groups and both sexes were involved in the study. Patients were divided in two groups as follow:
Group (I) SMD: Included 30 patients had submucosal diathermy of both inferior turbinates.

Group (II) PSIT: Included 30 patients had surgical endoscopic partial turbinectomy of both inferior turbinates.

Inclusion criteria: Patients with minimum 3 months duration of nasal obstruction combined with clinical finding of significant inferior turbinate hypertrophy and patients with mild deviated nasal septum.

Exclusion criteria: Active nasal or sinus infection, nasal polyp or enlarged obstructing adenoid, gross deviated nasal septum causing near total obstruction, septal perforation, bleeding disorders, patients with sino-nasal tumors or hypertrophic concha bullosa, patients refuse surgery or unfit for surgery and previous nasal operation.

All patients were subjected to full history taking (name, age, sex....), a detailed history of ear, nose and throat: with special emphasis on nasal symptoms (nasal obstruction, nasal discharge, sneezing and snoring). In addition, Full laboratory investigations were done before surgery such as CBC, ESR, Coagulation profile, hepatitis markers, blood glucose level, renal function tests and liver function tests to exclude any cases unfit for surgery. Besides, nasal endoscopy ( $2.7 \mathrm{~mm}$ and $4 \mathrm{~mm}$ diameter, $0^{\circ}$ nasal endoscope, Karl Storz, Germany) was used the endoscope without the use of local decongestants to assess the actual turbinate size pre and postoperatively according to the grading system described. Computed Tomography (CT) was done for all cases, it was performed for each patient in coronal and axial views with the use of local decongestants 10 minutes before the $\mathrm{CT}$ examination.

\section{Surgical procedures:}

Group (I) had turbinate reduction through submucosal diathermic coagulation of the inferior turbinate and group (II) had turbinate reduction through partial resection of bone and soft tissue of inferior turbinate.

1) Sub-mucosa diathermic coagulation of hypertrophied inferior turbinate.

Diathermic cautery was performed using an insulated needle electrode. The needle tip was pressed against the anterior end of the inferior 
turbinate and activated for a short period giving a devascularized zone to reduce bleeding. The needle was then introduced into the sub-mucosa through this zone to the posterior end of the turbinate with special care to stay close to the bone. The mono-polar power diathermy is then turned on whilst the needle is slowly withdrawn over a period of 5 seconds. Three to five such passes were performed for each inferior turbinate at a coagulation current of $70 \mathrm{~W}$. If the diathermy current is sufficient, the mucosa of the turbinate blanches and shrinks. After both techniques of turbinate reduction, ribbon gauzes soaked in ephedrine (1:1000) were used to secure hemostasis during the procedure. Intra-nasal septal silastic splints were applied in both sides to prevent occurrence of adhesions between inferior turbinates and nasal septum. Final hemostasis was maintained by using a Merocel nasal pack (Medtronic, California, USA) which usually was removed after 48 hours. The septal splints were removed on the 10th postoperative day. All patients were received antibiotics in the form of cephalosporin (1 gm daily) and analgesics in the form of paracetamol $(500 \mathrm{mg}$ three times per day) for 7-10 days postoperatively, also patients were instructed to use local nasal decongestants and nasal douche with sodium bicarbonate for 2 weeks postoperatively.

2) Partial surgical inferior turbinectomy (PSIT).

The inferior turbinate was infiltrated with ephedrine $(1: 1000)$ up to the posterior end. The

Table (1): NOSE Scale.

1. Nasal congestion or stuffiness

2. Nasal blockage or obstruction

3. Trouble breathing through my nose

4. Trouble sleeping

5. Unable to get enough air through my nose during exercise or exertion

Statistical analysis:

Data were statistically analyzed using SPSS software computer package (Statistical Package for Social Science) version 21 (SPSS Inc, USA). Graphics were done by Excel Microsoft inferior turbinates were mediatized using a blunt freer type of turbinate elevator then mucosa was crushed at its attachment to lateral nasal wall using an intestinal clamp forceps. Using the turbinectomy scissors, the bulk of the anterior and midportion of the inferior turbinate was removed medial to the crush portion. Posterior end of the inferior turbinate was removed with a special scissor which crushes and then cuts the tissue ${ }^{(16)}$.

\section{Postoperative follow up:}

In each visit we compared the two groups regarding the improvement of nasal obstruction using the (NOSE) scale (table 1). American academy of otolaryngology commissioned a study to develop a disease specific outcomes instrument for nasal obstruction, known as the nasal obstruction symptom evaluation (NOSE) scale. The nasal obstruction symptom evaluation scale (NOSE scale) is a valid, reliable and responsive instrument that is brief and easy to complete and has potential use for outcomes of studies in adults with nasal obstruction. Basing on (NOSE) scale we graded nasal obstruction of patients into mild, moderate and sever ${ }^{(17)}$.

Nasal obstruction was analyzed according to (NOSE) scale by asking the patients to score relief of nasal obstruction pre and postoperatively from 0-100 and was categorized as follow: Mild obstruction (5-25\%), moderate obstruction (30-50\%), sever obstruction (55$75 \%)$ and extreme obstruction (80-100\%).

\section{Nota Very Mild Moderate Fairly Bad Severe Problem Problem problem Problem problem}

0

0

0

0

0

1
1
1
1
1

$\begin{array}{lll}2 & 3 & 4 \\ 2 & 3 & 4 \\ 2 & 3 & 4 \\ 2 & 3 & 4 \\ 2 & 3 & 4\end{array}$

office 2010. Qualitative data were presented as frequency and percent however, quantitative data were presented as mean \pm standard deviation (SD) and sometimes, minimum and 
maximum were calculated (range). For comparison between means, the students $(\mathrm{T})$ test was used and Chi square test $\left(\chi^{2}\right)$ was used for comparisons in qualitative data. One way ANOVA was used to compare among different periods of test. Statistical significance was considered at $\mathrm{p}$. value $<0.05$. P-value was considered to be significance if less than 0.05 , high significance if less than 0.01 , or insignificant if more than 0.05 .

\section{RESULTS}

The results of this study showed that there was no significant difference between groups as regards age and sex distribution.

Table (2) presents the results of the comparison between groups as regard nasal score mean. The results showed that there were no significant differences between groups in nasal score mean at pre-operative, 1 week and 1 month (postoperative), however, endoscopic partial turbinectomy group (group, II) had significantly $(p<0.01)$ lower nasal score mean at 6 months compared to Submucosal diathermy group (group, I), (8.7 vs. 39.6\%). By another side, in submucosal diathermy group (group, I), nasal score recorded a significant decrease (improvement in nasal obstruction) at 1 week $(48.7 \%)$ and 1 month $(27.8 \%)$ post operative, however, it increased significantly to (39.6\%) at 6 months. However, in endoscopic partial turbinectomy group (group, II), nasal score recorded a significant decrease at 1 week (49.3\%), 1 month (25.8\%) and 6 months (8.7\%) post operative, respectively. This means that group (I) had a significant type of recurrence compared to group (II), (fig. 1).

The results of table (2) revealed that no significant differences were found between groups at pre-operative, 1 week and 1 month (post-operative), while, a significant improvement was found at 6 months in endoscopic partial turbinectomy group "which did not have any cases with moderate, severe or extreme obstruction compared to Submucosal diathermy group, which had 25 cases, $83.3 \%$ with moderate obstruction and 1 case, $3.3 \%$ with severe obstruction. In addition, the results showed that nasal obstruction was improved significantly in endoscopic partial turbinectomy group along with time than in Submucosal diathermy group which had high recurrence at 6 months post-operative.

Table (2): Nasal score mean between groups and among different period of test.

\begin{tabular}{lccc}
\hline \multicolumn{1}{c}{ Nasal score } & $\begin{array}{c}\text { Group (I) } \\
\text { Submucosal diathermy } \\
(\mathbf{n = 3 0})\end{array}$ & $\begin{array}{c}\text { Group (II) } \\
\text { Endoscopic partial } \\
\text { turbinectomy } \\
(\mathbf{n}=\mathbf{3 0})\end{array}$ & $\begin{array}{c}\text { P. value } \\
\text { (Sig.) }\end{array}$ \\
\hline Pre-operative & $68.3^{\mathbf{a}} \pm 16.3$ & $71.3^{\mathbf{a}} \pm 15.1$ & $0.451^{\mathrm{NS}}$ \\
$\mathbf{1}$ week & $48.7^{\mathbf{b}} \pm 13.6$ & $49.3^{\mathbf{b}} \pm 10.2$ & $0.847^{\mathrm{NS}}$ \\
$\mathbf{1}$ Month & $27.8^{\mathbf{d}} \pm 11.8$ & $25.8^{\mathbf{c}} \pm 9.9$ & $0.472^{\mathrm{NS}}$ \\
6 Months & $39.6^{\mathbf{c}} \pm 9.6$ & $8.7^{\mathbf{d}} \pm 4.8$ & $<\mathbf{0 . 0 0 1}^{* *}$ \\
\multicolumn{1}{c}{ P. value (Sig.) } & $\mathbf{< 0 . 0 0 1}^{* *}$ & $\mathbf{< 0 . 0 0 1}^{* *}$ & - \\
\hline
\end{tabular}

One way ANOVA was used to compare among different periods of test

T-tests was used to compare between groups .

a, b, c, d Means in the same column (group) with different superscript are significantly different.

NS Not significant $\quad *$ Significant $(\mathrm{p}<0.05) . \quad * *$ Highly significant $(\mathrm{p}<0.01)$.

Table (3): Nasal score grades at pre-operative, 1 week, 1 month, 6 months post-operative. 


\begin{tabular}{|c|c|c|c|c|}
\hline \multicolumn{2}{|c|}{ Nasal score grades } & \multirow{2}{*}{ 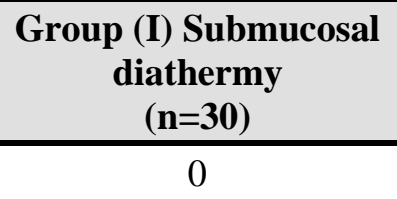 } & \multirow{2}{*}{$\begin{array}{c}\begin{array}{c}\text { Group (II) Endoscopic } \\
\text { partial turbinectomy } \\
(\mathbf{n}=\mathbf{3 0})\end{array} \\
0\end{array}$} & \multirow{2}{*}{$\begin{array}{c}\begin{array}{c}\text { P. value } \\
\text { (Sig.) }\end{array} \\
0.699^{\mathrm{NS}}\end{array}$} \\
\hline Pre- & Mild & & & \\
\hline operative & Moderate & $4(13.3 \%)$ & $2(6.7 \%)$ & \\
\hline & Severe & $15(50.0 \%)$ & $13(43.3 \%)$ & \\
\hline & Extreme & $11(36.7 \%)$ & $15(50.0 \%)$ & \\
\hline \multirow[t]{4}{*}{1 Week } & Mild & $2(6.7 \%)$ & $1(3.3 \%)$ & $0.536^{\mathrm{NS}}$ \\
\hline & Moderate & $18(60.0 \%)$ & $16(53.4 \%)$ & \\
\hline & Severe & $9(30.0 \%)$ & $13(43.3 \%)$ & \\
\hline & Extreme & $1(3.3 \%)$ & 0 & \\
\hline \multirow[t]{4}{*}{1 Month } & Mild & $18(60.0 \%)$ & $20(66.7 \%)$ & $0.764^{\mathrm{NS}}$ \\
\hline & Moderate & $11(36.7 \%)$ & $10(33.3 \%)$ & \\
\hline & Severe & $1(3.3 \%)$ & 0 & \\
\hline & Extreme & 0 & 0 & \\
\hline \multirow[t]{5}{*}{6 Months } & No symptoms & 0 & $3(10.0 \%)$ & $<0.001 * *$ \\
\hline & Mild & $4(13.3 \%)$ & $27(90.0 \%)$ & \\
\hline & Moderate & $25(83.3 \%)$ & 0 & \\
\hline & Severe & $1(3.3 \%)$ & 0 & \\
\hline & Extreme & 0 & 0 & \\
\hline
\end{tabular}

Chi-square tests was used to compare between groups .

Table (4) showed the nasal score grades in submucosal diathermy (group, I) during different time of test. The results showed that there was a significant improvement in nasal obstruction along with time evidenced by the significant decrease in cases with extreme, severe and moderate obstruction at 1 week, 1 month post-operative (to be in mild grade). However, a type of recurrence was happened at 6 months (post-operative) where 14 cases $(46.6 \%)$ had moderate obstruction after they were at mild obstruction grade at 1 month (fig. 2).

Results of table (5) presents nasal score grades in endoscopic partial turbinectomy (group, II) during different time of test. The results showed that there was a significant decrease in nasal score (improvement in nasal obstruction) along with time evidenced by the significant decrease in cases with extreme, severe and moderate obstruction at 1 week, 1 month and 6 months, respectively with no recurrence .Preoperatively, $50 \%$ of cases had extreme obstruction and $43.3 \%$ of them had severe one and 27 cases $(90.0 \%)$ were significantly improved and had mild obstruction. However, 3 cases $(10.0 \%)$ had no symptoms at 6 months post-operatively (fig. 3 ).

Table (4): Nasal score grades in submucosal diathermy (group, I) during different time of test.

\begin{tabular}{lccccc}
\hline \multirow{2}{*}{ Nasal score grades } & \multicolumn{4}{c}{ Time of test } \\
Pre-operative & 1 Week & 1 Month & 6 Months & P. value \\
(Sig.)
\end{tabular}

Table (5): Nasal score grades in endoscopic partial turbinectomy (group, II) during different time of test. 


\begin{tabular}{lccccc}
\hline \multirow{2}{*}{ Nasal score grades } & Pre-operative & 1 Week & 1 Month & 6 Months & $\begin{array}{c}\text { P. value } \\
\text { (Sig.) }\end{array}$ \\
\hline No symptoms & 0 & 0 & 0 & $3(10.0 \%)$ & $<\mathbf{0 . 0 0 1 * *}$ \\
Mild & 0 & $1(3.3 \%)$ & $20(66.7 \%)$ & $27(90.0 \%)$ & \\
Moderate & $2(6.7 \%)$ & $16(53.4 \%)$ & $10(33.3 \%)$ & 0 & \\
Severe & $13(43.3 \%)$ & $13(43.3 \%)$ & 0 & 0 & \\
Extreme & $15(50.0 \%)$ & 0 & 0 & 0 & \\
\hline
\end{tabular}

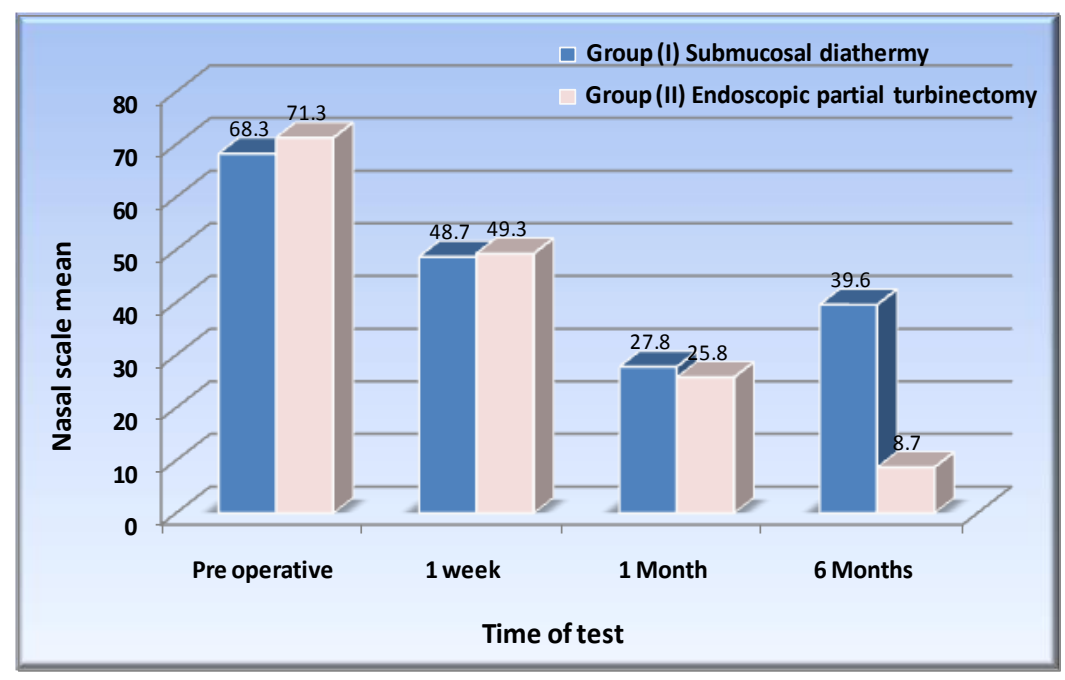

Figure (1): Nasal score mean between groups at different times of test.

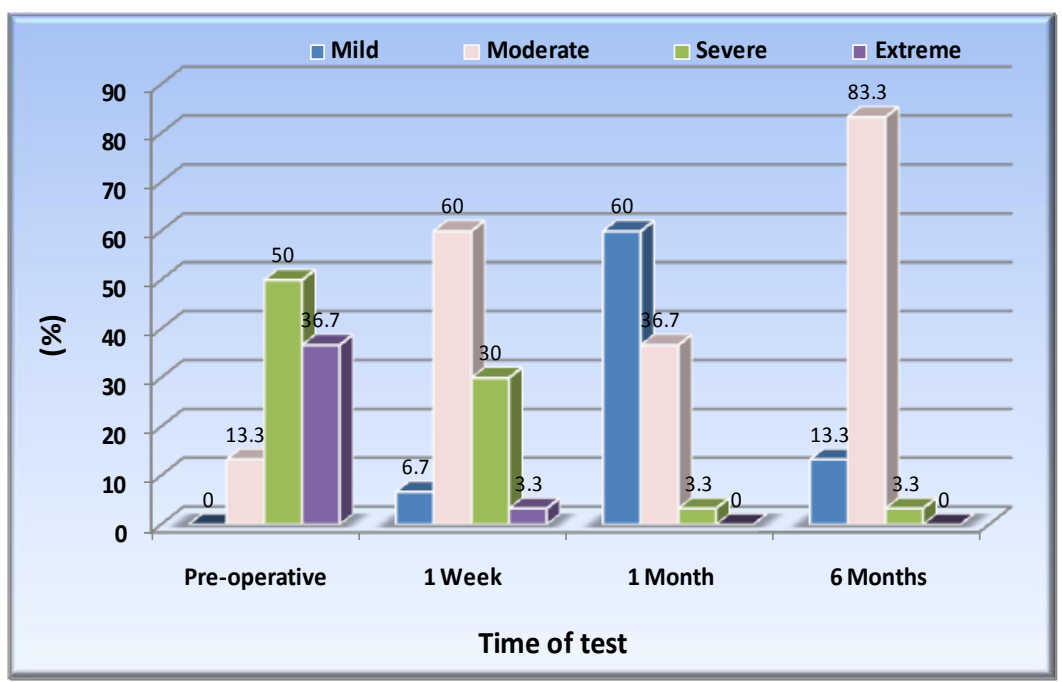

Figure (2): Nasal score grades of group (I) during different times of test. 


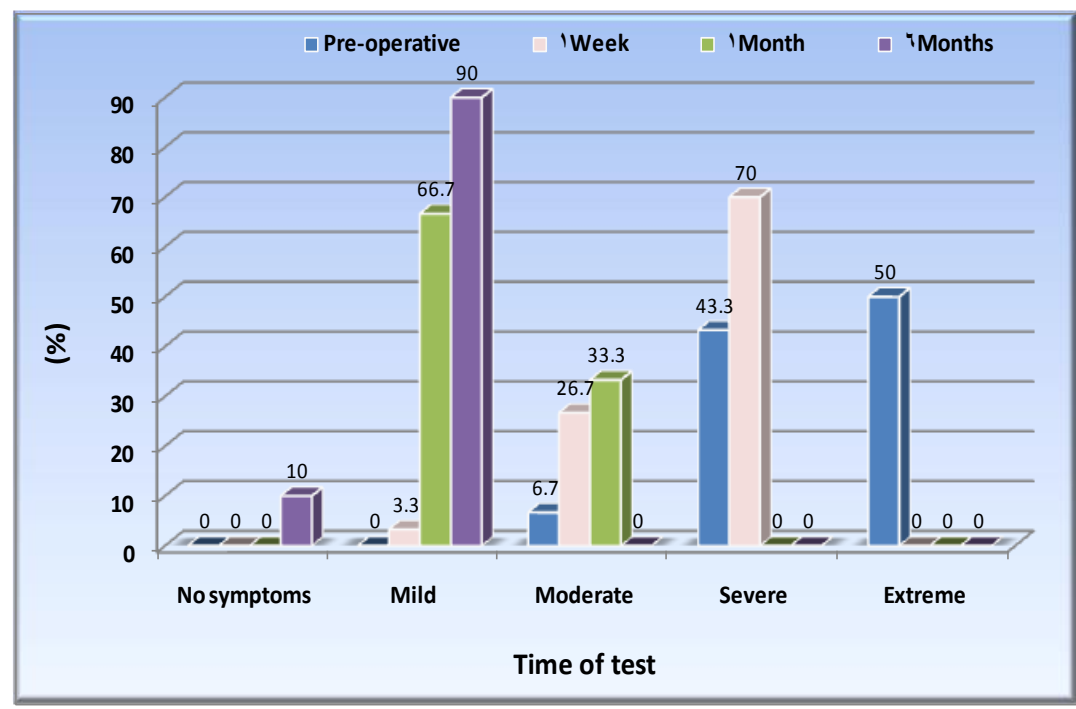

Figure (3): Nasal score grades of group (II) during times of test.

\section{DISCUSSION}

Chronic nasal obstruction is a common subjective complaint seen by otolaryngologists and it is usually caused by either swelling of the nasal tissue or an anatomical blockage which results in a narrowing of the nasal cavity and the feeling of the passages being congested and can lead to sleeping disorders, snoring and obstructive sleep apnea ${ }^{(18)}$. Nasal obstruction can be caused by a deviated nasal septum, enlarged turbinates, nasal polyps, enlarged adenoids, tumors and nasal congestion ${ }^{(19)}$. The goal of turbinate reduction surgery is to improve nasal breathing and reduce nasal drainage and post-nasal drip which can improve the patient's quality of life by decreasing headaches, snoring and sleep apnea ${ }^{(20)}$.

The preset results revealed that there were no significant differences between groups regarding mean age and sex distribution. These results also agreed with Smitha ${ }^{(21)}$ and Gomma (16) who compared both techniques used in our study and did not find any significant differences between groups regarding age and sex distribution. To our knowledge, this is important to ensure the homogenization of the studied groups to get accurate results from the comparison between groups and to eliminate bias. In addition, Nawaz ${ }^{(19)}$ studied the nasal patency following sub mucosal diathermy (SMD) of inferior turbinate and inferior turbinectomy (IT), they found that both the groups are comparable with respect to age and gender.
In this study we used the NOSE scale (score) for the evaluation of the degree of nasal obstruction in our study. The results showed that there were a significant post-operative improvement in nasal obstruction in both used techniques (submucosal diathermy and endoscopic partial turbinectomy) at 1 week and 1 month. However, this significant improvement continued to 6 months in endoscopic partial turbinectomy group (preoperatively, $50 \%$ of cases had extreme obstruction and $43.3 \%$ of them had severe one and 27 cases $(90.0 \%)$ were significantly improved and had mild obstruction. However, 3 cases $(10.0 \%)$ had no symptoms at 6 months post-operatively "100\% improvement"). While, a type of recurrence was happened in submucosal diathermy group at 6 months (postoperative), 14 cases of them (46.6\%) had moderate obstruction after they were at mild obstruction grade at 1 month. Our results agreed with those of Datta ${ }^{(10)}$ who evaluated the efficacy of submucosal diathermy and partial inferior turbinectomy in the treatment of hypertrophied inferior turbinate. They found that both techniques were found to be effective in reducing nasal obstruction. Also, similar to our findings, they found that there were 5 cases with recurrence of nasal obstruction from no symptom to mild symptom at 6 months in submucosal diathermy group while no cases of recurrence were observed in the partial inferior 
turbinectomy group at 6 months indicating better effectiveness on long term.

The present results also are similar with Smitha ${ }^{(21)}$ who analyzed and evaluated the efficacy of sub mucosal diathermy and partial inferior turbinectomy in the treatment of symptomatic hypertrophied inferior turbinates in allergic rhinitis patients. They found that in submucosal diathermy group, significant improvement of nasal airflow was seen in $14 \%$ of patients \& $85.9 \%$ had moderate improvement. In partial inferior turbinectomy group, $41.3 \%$ of patients had significant improvement \& $58.6 \%$ had moderate improvement. They concluded that partial inferior turbinectomy was found to be more effective in relieving nasal obstructionin allergic rhinitis patients for longer duration and with no major complications. In addition, Nawaz ${ }^{(19)}$ found that all the patients in both groups (submucosal diathermy (group, A) and partial inferior turbinectomy (group, B)) had severe nasal obstruction pre-operatively. Three weeks post operatively in group A, 20 (25\%) patients had no obstructive symptoms, 36 (45\%) had mild obstructive symptoms while 24 $(30 \%)$ had moderate nasal obstruction. While in group B, $58(72.5 \%)$ felt complete relief with no-obstruction, $22(27.5 \%)$ patients had mild obstruction while none had moderate or severe obstruction of nose. Post-operative nasal patency was significantly better in group B as compared to group A $(\mathrm{p}<0.001)$.

In a study by Kafle (22), they compared effectiveness of sub mucosal diathermy and partial resection of inferior turbinate. Following six months of follow up, six patients of group undergoing SMD had recurrence with nasal blockage and in patients undergoing inferior turbinectomy none had recurrence. It was concluded that partial resection of inferior turbinates is better than SMD in long course. In the same line to our findings, Khosa ${ }^{(23)}$ studied the outcome of SMD and partial inferior turbinectomy in patients with chronic hypertrophic rhinitis in terms of relief of nasal obstruction. They found that both these procedures are simple and easy to perform, SMD leads to a dramatic fall in nasal obstruction but the patient do not have concurrent medical treatment, inferior turbinate re-hypertrophy within 15 months. They concluded that both the procedures are relatively safe and effective and do not need expensive instrumentation.
All surgical methods aim mainly in reducing the size of turbinate to relieve nasal obstruction, but few studies have shown that surgical methods are known to reduce nasal discharge, improve hyposmia and decrease the attacks of asthma and sneezing ${ }^{(24)}$. Anil and Bilkhis ${ }^{(18)}$ studied the comparison between partial inferior turbinectomy and submucosal diathermy in the management of inferior turbinate hypertrophy. They found that partial inferior turbinectomy showed better and early results when compared to submucosal diathermy in terms of subjective improvement of symptoms. In addition, patients who underwent partial inferior turbinectomy showed marked improvement of nasal obstruction at the end of first week itself with $80 \%$ of them having total improvement while the remaining $20 \%$ said that the nasal obstruction was reduced to being a mild problem. At the end of second month, 49 patients [98\%] had no nasal obstruction while only one patient said that it remained as a mild problem. They concluded that early and better results were seen in patients who underwent partial inferior turbinectomy.

Recently, Vishnu and Rajamma ${ }^{(25)}$ evaluated and analyzed the impact of partial turbinectomy and SMD on nasal obstruction and to compare the results of either procedures in respect of safety and efficacy. They found that SMD is relatively safe and less invasive than partial turbinectomy but its effectiveness compared to partial turbinectomy is short lived. In the long run, partial turbinectomy was found to be more effective. On the other hand, in a study by Gomma (16) showed that both techniques (submucosal diathermy and partial inferior turbinectomy) were equally effective in improving of nasal obstruction and degree of tissue healing.

The present results showed that there was a significant improvement in nasal obstruction along with time evidenced by the significant decrease in cases with extreme, severe and moderate obstruction at 1 week, 1 month postoperative (to be in mild grade). However, a type of recurrence was happened at 6 months where 14 cases $(46.6 \%)$ had moderate obstruction after they were at mild obstruction grade at 1 month. Similar success rates were observed by Fradis ${ }^{(26)}$, where nasal breathing had improved post submucosal diathermy by $89 \%$. In addition, Farmer and Eccles, ${ }^{(27)}$ reported a good outcome of 412 patients undergoing submucosal diathermy. In addition, another 
study by Fradis ${ }^{(26)}$ showed $78 \%$ success rate at one year postoperatively. In addition, $84 \%$ of success rate was observed by Ashoor ${ }^{(28)}$ at end of 1 month postoperatively.

The effect of submucous diathermy was achieved through coagulation of the venous sinusoids within the turbinate, leading to submucosal fibrosis ${ }^{(13)}$. Although turbinate tissue volume reduction by various techniques leads to shrinkage of the turbinate size, however the epithelial changes of chronic hypertrophic turbinate remains more or less unaltered ${ }^{(2)}$. Our results are supported by Smitha ${ }^{(21)}$ who found that partial inferior turbinectomy was found to be more effective in reducing the nasal obstruction in all 58 patients. At 3rd month 27 patients $(46.55 \%)$ had complete relief from nasal obstruction \& $53.4 \%$ of patients had moderate improvement. At 6th month 24 patients $(41.34 \%)$ had complete relief from nasal obstruction \& 43 patients $(58.6 \%)$ had moderate improvement. In addition, this was in accordance with other studies by Yoseph ${ }^{(29)}$ who showed $87 \%$ improvement in nasal obstruction at 1 year $\& 77 \%$ rate at $2-5$ years. In addition, $75 \%$ success rate was observed in the study by Elwany and Harrison ${ }^{(30)}$.

Ophir ${ }^{(31)}$ studied the effects of total inferior turbinectomy in one hundred and fifty patients. He found that $91 \%$ of patients had patent nasal airway post operatively; they showed $82 \%$ success even after 10-15 years. A study involving 357 patients was conducted at Department of Otolaryngology-Head and Neck Surgery, Western Galilee Hospital, Nahariya which showed that even in a hot and dusty climate, total inferior turbinectomy was an effective and relatively safe procedure in relieving nasal obstruction. In addition, the results of inferior turbinectomy in our study are in accordance with the above mentioned international studies. Besides, Khan ${ }^{(32)}$ studied post operative results in 135 patients who underwent inferior turbinectomy for hypertrophied inferior turbinates. They found that 128 patients reported marked improvement in nasal breathing, four had mild improvement, and three had no change. Also, it was found that total inferior turbinectomy is safe and very effective in relieving chronic nasal obstruction caused by hypertrophied inferior turbinates and did not require expensive instrumentation. A study was also performed on one hundred and sixteen patients in which inferior turbinectomy was found to be effective in controlling the symptoms of $94 \%$ patients with inferior turbinate hypertrophy ${ }^{(28)}$.

There were various studies, which showed that partial inferior turbinectomy is as effective procedure in relieving nasal obstruction as total inferior turbinectomy with success rate ranging from 70 to $80 \%{ }^{(18)}$. However partial inferior turbinectomy should be performed cautiously in order to protect anatomical structures and physiological functions of nose Monopolar diathermy is an old technique for the reduction of sub-mucosal tissue of the inferior turbinate, but still widely practiced ${ }^{(16)}$.

This study had some limitations, of them: the relative small sample size and that we could not involve some investigated variables such as assessment of nasal pain, post-operative bleeding and complications.

\section{CONCLUSIONS}

Based on our findings, endoscopic partial turbinectomy caused a significant improvement in nasal obstruction at 6 months postoperative compared to submucosal diathermy. Submucosal diathermy had high recurrence at 6 months post-operative, however, there was no recurrence in endoscopic partial turbinectomy. Both of the used surgical techniques were found to be effective in reducing nasal obstruction but endoscopic partial turbinectomy was more effective in reducing nasal obstruction for longer duration.Further prospective studies are required with larger sample size and longer follow up period.

Ethical considerations: Ethical permission was obtained from a Local Research Ethics Committee (REC) of Faculty of Medicine, Alazhar University and an informed consent was taken from all patients before enrolment in the study.

\section{REFERENCES}

1- Dayal A, John S R, Guilherme J M G (2016): Impact of Middle vs. Inferior Total Turbinectomy on Nasal Aerodynamics. Otolaryngol Head Neck Surg., 155(3): 518-525. 2- Deenadayal $D S$, M Naveen $k, P$ Sudhakshin, Saif H (2014): Radiofrequency Reduction of Inferior Turbinates in Allergic and Non Allergic Rhinitis. Indian J Otolaryngol Head Neck Surg., 66 (1): 231-236. 
3- $\quad$ Erickson B, Robert H, Caroline J et al. ( 2016): Acoustic rhinometry and video endoscopic scoring to evaluate postoperative outcomes in endonasal spreader graft surgery with septoplasty and turbinoplasty for nasal valve collapse. Journal of Otolaryngology - Head \& Neck Surgery, 45:2.

4- $\quad$ Wallace DV, Dykewicz MS, Bernstein DI et al. (2008): The diagnosis and management of rhinitis: an updated practice parameter. J Allergy Clin Immunol., 122:S1.

5- $\quad$ Scheithauer M O (2010): Surgery of the turbinates and "empty nose" syndrome. GMS Curr Top Otorhinolaryngol Head Neck Surg., 9: Doc03. 6- Cheng PW, Fang KM, Su HW et al. (2012): Improved objective outcomes and quality of life after adenotonsillectomy with inferior turbinate reduction in pediatric obstructive sleep apnea with inferior turbinate hypertrophy. Laryngoscope, 122(12):2850-2854.

7- $\quad$ Goyal PM, Hwang PH (2012): Surgery of the septum and turbinates. In: Kennedy DW, Hwang PH, (eds). Rhinology: Diseases of the Nose, Sinuses, and Skull Base. New York, NY: Thieme, pp. 444-456.

8- Sharhan SSA, Lee EJ, Hwang CS et al. (2018): Radiological comparison of inferior turbinate hypertrophy between allergic and nonallergic rhinitis: does allergy really augment turbinate hypertrophy? Eur Arch Otorhinolaryngol., 275 (4):923-9.

9- Singh DP, Forte AJ, Apostolides JG, Zahiri HR, Stromberg J, Alonso N et al. (2012): Transoral submucosal resection of the inferior turbinate: a novel approach to functional rhinoplasty. Ann Plast Surg., 68(1):46-8.

10- Datta R K, Ramya B, Vinay S R (2018): Comparative study between partial inferior turbinectomy and submucosal diathermy for treatment of inferior turbinate hypertrophy due to allergic rhinitis. Int J Otorhinolaryngol Head Neck Surg., 4(2):362-367.

11- Chhabra N, Houser SM (2011): The surgical management of allergic rhinitis. Otolaryngol Clin North Am., 44(3):779-95.

12- Poirier J, George C, Rotenberg B (2014): The effect of nasal surgery on nasal continuous positive airway pressure compliance. Laryngoscope, 124: 317-9.

13- Roje Z, Goran R and Goran K (2011): Efficacy and Safety of Inferior Turbinate Coblation-Channeling in the Treatment of Nasal Obstructions. Coll. Antropol., 35 1: 143-146

14- Ozcan KM, Gedikli Y, Ozcan I et al. (2008): Microdebrider for reduction of inferior turbinate: evaluation of effectiveness by computed tomography. Journal Otolaryngology Head and Neck Surgery, 37: 463-468.

15- Bhandarkar ND, Smith TL (2010): Outcomes of surgery for inferior turbinate hypertrophy. Curr Opin Otolaryngol Head Neck Surg., 18(1):49-53.

16- Gomaa MA, Abdel Nabi OG, Abdel Kerim ARA, Aly A (2015): Comparative Study between Partial Surgical Inferior Turbinectomy and Sub-mucosal Diathermy of Inferior Turbinate for Treatment of Inferior Turbinate Hypertrophy. Otolaryngol., 5:217.

17- Stewart MG, Smith TL, Weaver EM et al. (2004): Outcomes after nasal septoplasty: results from the Nasal Obstruction Septoplasty Effectiveness (NOSE) study. Otolaryngol Head Neck Surg.,130 (3):283-290.

18- Anil HT, Bilkhis MG (2014): Comparative Study between Partial Inferior Turbinectomy and Submucosal Diathermy in the Management of Inferior Turbinate Hypertrophy. International J Sci Res., 3(7):323-5.

19- Nawaz F, Arfat J, Sheraz B (2014): Comparison of nasal patency following submucosal diathermy of inferior turbinate versus inferior turbinectomy. Pak Armed Forces Med J., 64 (4):551-4

20- ENT institute (2015): Surgery Descriptions, Nasal and Sinus. http://www.entinstitute.com/surgery-descriptions/. 21- Smitha CBC, Kiran B, John S, Kumar $C$ (2015): Comparison of Partial Inferior Turbinectomy and Submucosal Diathermy for Hypertrophied Inferior Turbinate in Allergic Rhinitis Patients. J of Evolution of Med and Dent Sci.,4 (54):9457-67.

22- $\quad$ Kafle P , Maharjan $M$, Shrestha $S$, Toran KC (2007): Comparison of sub mucosal diathermy and partial resection of Inferior turbinate in the treatment of symptomatic nasal valve blockage .Kathmandu University Medical Journal, 5: 501-503

23- $\quad$ Khosa S H, Ali H S, Muhammad A N (2014): Role of Partial Inferior Turbinectomy in Patients with Chronic Hypertrophic Rhinitis. J M H S., 8: 3.

24- Mathai J (2004): Inferior turbinectomy for nasal obstruction review of 75 cases. Indian $\mathbf{J}$ Otolaryngol Head Neck Surg., 56(1):23-6.

25- Vishnu MS, Rajamma KB (2016): Comparison of Submucosal Diathermy and Partial Inferior Turbi nectomy. Int J Sci Stud.4 (1):120-3. 26- $\quad$ Fradis M, Malatskey S, Mangamsa I (2002): Effect of submucosal diathermy in chronic 
nasal obstruction due to turbinate enlargement. Am J Otolaryngol, 23: 332 -336.

27- $\quad$ Farmer S E, Eccles R (2006): Chronic inferior turbinate enlargement and the implications for surgical intervention. Rhinology, 44:234-238.

28- Ashoor A (2012): Efficacy of Submucosal Diathermy in Inferior Turbinate Hypertrophy. Bahrain Medical Bulletin, 34 (1): 145-158.
29- Yoseph R, Gabriel $R \quad$ (1996): Comparison of partial inferior turbinectomy \& cryosurgery of hypertrophic inferior turbinates. The Journal of Laryngology \& Otology, 110: 732735 .

30- Elwany S, Harison R (1990): Inferior turbinectomy: comparision of four techniques. The J Laryngol Otol.,104: 206-209.

31- Ophir D, Schindel D, Halperin D, Marshak G (1992): Long term follow up of the effectiveness \& safety of Inferior turbinectomy. Plas Reconstruction Surgery,980-984

32- Khan NU, Arshad M, Ahmed T, Ashfaq M (2005): Total Inferior Turbinectomy For Hypertrophied Inferior Turbinates: Postoperative Results In 135 Patients. Pak Armed Forces Med J., 55:187-92. 